\title{
Study of Modulation Schemes over a Multipath Fading Channels
}

\author{
Karthik Kumar Vaigandla ${ }^{1}$, Allanki Sanyasi Rao ${ }^{2}$, Kallepelli Srikanth ${ }^{3}$ \\ 1,2,3 Dept. of Electronics \& Communication Engineering, Balaji Institute of Technology and Science, Warangal, Telangana,India
}

To Cite this Article

Karthik Kumar Vaigandla, Allanki Sanyasi Rao and Kallepelli Srikanth. Study of Modulation Schemes over a Multipath Fading Channels. International Journal for Modern Trends in Science and Technology 2021, 7 pp. 34-39. https://doi.org/10.46501/IJMTST0710005

\section{Article Info}

Received: 02 September 2021; Accepted: 28 September 2021; Published: 30 September 2021

\section{ABSTRACT}

Communications systems concerted over wireless channels depend on the environment. Communications system can be more reliable and efficient by properly analyzing wireless channels. Today's most important features are a high data rate and reliable performance to exploiting viable networks during this new information age. The channel is not time-invariant in wireless communication, so the received signal exhibits amplitude, phase, and angle variations due to multipath fading. Increasing data rates and reducing bandwidth make Orthogonal Frequency Division Multiplexing (OFDM) an important component of wireless communication systems. The OFDM technique uses many carriers very efficiently. With this scheme, interference is robustly reduced, and fading scenarios are easily accommodated. Analyzing digital modulation schemes requires evaluating link performance with fading channels. The paper compares channel performance over varying fading environments using a variety of modulation schemes. We study the BER and SNR properties of the AWGN, Rician fading and Rayleigh fading channels modulated with BPSK, QPSK, and M-ary QAM.

KEYWORDS: Additive White Gaussian Noise (AWGN), Bit Error Rate (BER), Frequency selective fading, M-Ary Modulation Scheme, Multiple Input Multiple Output (MIMO), Orthogonal Frequency Division Multiplexing (OFDM), Rayleigh and Rician fading channel, Symbol Error Rate (SER), Signal to Noise Ratio (SNR).

\section{INTRODUCTION}

Nowadays, as wireless multimedia communications become more and more popular, faster bit rates and greater reliability are expected to be a major requirement [2]. By using OFDM, we are able to achieve Communication at high speed and high spectral efficiency, similarly time accommodating more users [9]. The system supports next-generation wireless technology by providing multiplexing/multiple access technologies [10]. The property of orthogonality enables, a common channel is used to send several signals without interfering with each other, so that an interference-free detection can take place. By losing orthogonality, information signals become blurred and low-quality transmission is hampered.

A high throughput and speed are highly recommended in wireless communication nowadays. Since the high-speed wired communications became popular decades ago, wireless communication has grown in popularity. Wireless networks have used OFDM to communicate downlink. The channels are easily adapted to the difficult channel conditions. Wireless communications using OFDM are an excellent choice for 4G. However, OFDM's PAPR is its main disadvantage. There are several methods for reducing the PAPR [25]. 
Signal fading can be caused by reflections or obstructions on the path between transmitter and receiver. Each of the rays arriving at the receiver has a slight delay and gains that are different, so signals reach the receiver in this situation via a variety of directions. Phase shifts are caused by the time delay, the signal degradation and leads to fading that renders the signal nearly inaudible. When a signal occurs in an environment with a maximum time delay, the delay spread is measured [2].

An antenna becomes aware of two or more paths for receiving a signal as a result of multipath transmission in wireless telecommunications. A multipath signal includes the reflection of the ionosphere, ducting, reflection of water bodies, and reflection of objects such as mountains. As a result of multipath, signals are shifted in phase and interfere constructively and destructively. Communication quality can be affected by multipath propagation in digital radio. A receiver or transmitter's effect, as well as surrounding disorder, is included [8].

Mobile/wireless communications suffer most from fading and interference. Simulation and modeling of communication systems under fading channels are essential for improving and testing the system's resistance to fading in order to achieve optimum performance. Fading channels have diverse characteristics that depend on the propagation environment. Therefore, in this regard, it is essential to design proper fading models based on the communication context [1].

In order to measure the quality of communication, SNR and bit BER are commonly used. The SNR measures how much noise there is in comparison to the signal. It is possible to use SNR to predict how well the system will perform in terms of reception accuracy. In BER, a digital bit is classified as erroneous or not based on simple binary comparison. Modern communications systems measure performance based on BER versus SNR [6]. In wireless communication, the most commonly used fading models are AWGN, Rayleigh fading, and Rician fading. In this paper, different modulation schemes are compared in order to evaluate their performance using these channels.

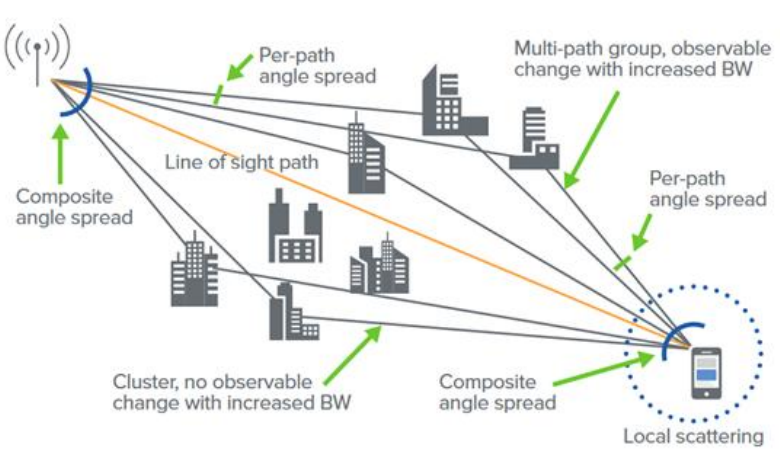

Figure 1. Wireless Propagation Environment

\section{SYSTEM MODEL}

\section{A.Orthogonal Frequency Division Multiplexing (OFDM)}

OFDM is a more efficient and time-efficient $5 \mathrm{G}$ modeling technique. OFDM has a high PAPR, which is its major disadvantage. Thus, the nonlinear gadgets could be detected by the OFDM receiver [19]. This figure 2 illustrates the basic block diagrams of OFDM communications systems. There are $\mathrm{N}$ subcarriers in the OFDM signal, each modulated by a complex symbol selected out of a PSK/QAM constellation [11]. M-QAM is used to modulate the input data stream. After that, a parallel-to-serial conversion was carried out on the symbol stream. Each of the $\mathrm{N}$ subcarriers is modulated with a set of $\mathrm{N}$ parallel symbols, which is output by $\mathrm{S} / \mathrm{P}$. OFDM modulators have discrete frequency components. Through the use of IFFT, which consists of sequences of OFDM symbols of length $\mathrm{N}$, this output is converted into time samples [6]. $\mathrm{N}$ can be represented by the following vector complex: $X=\left[X_{0}, X_{1}, X_{2}, X_{3}, \ldots\right.$, $\mathrm{X}_{\mathrm{N}-1}$. An OFDM signal with subcarriers and complex baseband can be written as :

$$
x(m)=\frac{1}{\sqrt{N}} \sum_{i=0}^{N-1} X(i) e^{\frac{j 2 \Pi i n}{N}} ; i=0,1, \ldots, N-1
$$

On the assumption that carrier frequency and symbol timing are perfectly synched, demodulation is performed. In frequency domain, the use of $\mathrm{CP}$ eliminates inter-block interference, so each OFDM block is handled separately, there is a delay spread on the channel equal to or greater than the length of the CP. At the output of amplifiers, samples are generated according to $\mathrm{y}=\left[\mathrm{y}_{0}, \mathrm{y}_{1}, \ldots ., \mathrm{y}_{\mathrm{N}-1}\right]$. 

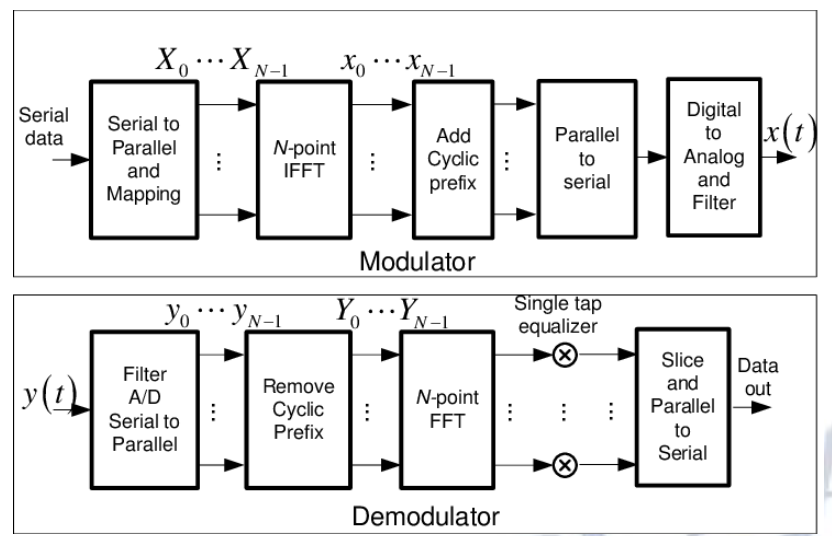

Figure 2. OFDM Block Diagram

\section{B. Multiple Input Multiple Output (MIMO)}

Wireless communications have increasingly embraced MIMO antenna systems. Multi-antenna systems improve both capacity and performance over single antennas by implementing each end of the link has multiple antennas [24]. In MIMO, the algorithms use narrowband. Wideband wireless channels have a frequency selective nature, which can be mitigated by combining MIMO with OFDM. Through OFDM, a frequency selective channel is effectively divided into a number of parallel flat fading sub channels. MIMO and OFDM are thus complementary. The MIMO algorithms are able to operate in broadband communication by transmitting and detecting each subcarrier simultaneously [20].

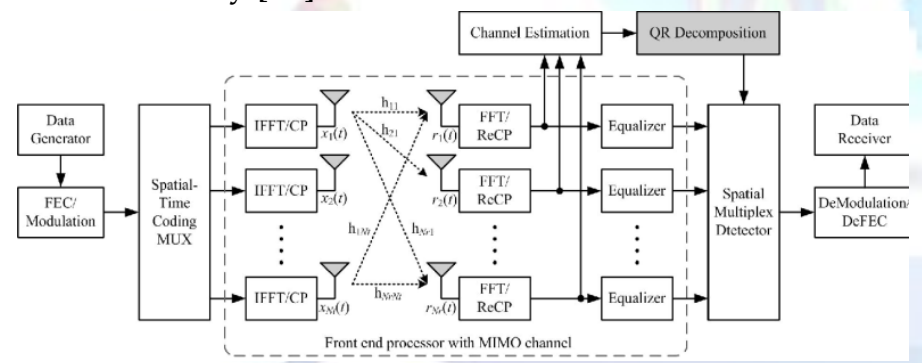

Figure 3. MIMO-OFDM

$\mathrm{N}_{\mathrm{t}}$ transmitters and $\mathrm{N}_{\mathrm{r}}$ receivers antenna configuration is shown in Figure 3. The OFDM protocol adds another dimension to MIMO, namely, the frequency dimension, to exploit in addition to the spatial and temporal dimensions. Space-Time-Frequency (STF) mappers enable the encoded bits to be mapped between the three available dimensions based on the one-dimensional input bit stream [21]. The STF mapper almost fully reveals an OFDM transmitter. FFT is performed separately for each receiver branch at receiver side, eliminating the CP. The STF must be detected and decoded in order to retrieve the binary data stream [22]. It is common for MIMO algorithms to use single carriers; MIMO detection is done using OFDM subcarriers. In OFDM, there is a degree of parallelism by means of subcarriers that enhances the performance. Utilizing the MIMO-OFDM technique, it will enable better communication [23].

\section{BER AND SNR PERFORMANCE ANALYSIS FOR VARIOUS FADING CHANNELS}

\section{A. Quadrature Phase Shift Keying (QPSK)}

The QPSK method modulates two bits at the same time, selectively shifting a carrier to one of four possible planes (0, 90, 180, 270 degrees). Signals carrying QPSK information use the same bandwidth as traditional PSK signals. Video transmissions over satellites, cable modems, videoconferences, cellular phones and other digital communication over radio waves are all done in QPSK [14]. In comparison with BPSK modulation, this modulation scheme doubles the data rate while maintaining the same bandwidth. BPSK has the advantage of being simple, while QPSK requires the use of more complicated transmitters and receivers [15]. QPSK is defined by the equation:

$$
S_{k}(t)=\sqrt{\frac{2 e_{b}}{T_{b}}} \cos \left[2 \Pi f_{c} t+\frac{(2 k-1) \Pi}{4}\right] ; k=0,1,2,3
$$

BER for QPSK : Two binary phase shift keying is used to define the QPSK modulation of a signal [1]. Probability of bit errors is $P_{b}=Q\left[\sqrt{2 \gamma_{b}}\right]$ an error probability of a symbol (SER) is $P_{s}=1-\left\{1-Q\left[\sqrt{2 \gamma_{b}}\right]\right\}^{2}$. It consists of two equal channels with the same bit rate per bit of the symbol $\gamma_{s}=2 \gamma_{b}=\frac{A^{2}}{N_{o}}$. Hence SER is $P_{s} \leq \frac{3 e^{-0.5 \gamma_{s}}}{\sqrt{2 \Pi \gamma_{s}}}$.

SER for M-PSK : For MPSK signaling, it is simple to calculate the SER using nearest neighbor approximation. SER is $P_{s} \approx 2 Q\left[\sqrt{2 \gamma_{s}} \operatorname{Sin}(\Pi / M)\right]$ 


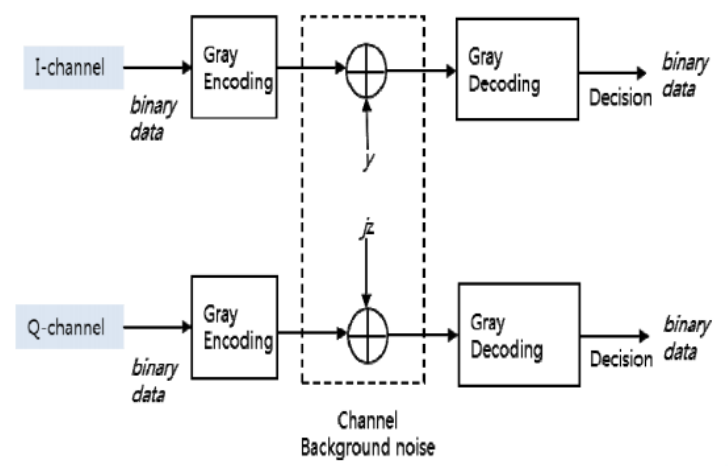

Figure 4. System model for QPSK

\section{B. Quadrature Amplitude Modulation (QAM)}

QAM uses two amplitude modulated carriers in phase with each other at an angle of 90 degrees. The efficient transmission of information is improved by mixing phase changes with amplitude changes. Compared to conventional time/amplitude modulated schemes, this scheme can carry higher data rates. As the modulation format indicates, $\mathrm{N}$ point constellation, this refers to the number of points approximately at which the signal can rest during transmission [16].

SER for QAM : Two M-PAMs on in phase and quadrature components are used in the SER for $M-Q A M$ with $M=2^{N}$. Using each branch/channel of M-PAM, we can find out the error probability for QAM modulation. An approximation of SER for high SNR is $P_{s} \approx 4 Q\left[\sqrt{\frac{3 \gamma_{s}}{M-1}}\right]$.

\section{Binary Phase Shift Keying (BPSK)}

The BPSK scheme is a means of modulating or changing the phase of a reference signal, it is simplest type of phase shift keying [12]. The constellation points used in this method are arranged around a circle with uniform spacing around each phase. Phase separation between adjacent points is maximized by using this method, so there is no corruption potential between adjacent points. The energy is transmitted with equal power since they are positioned on a circle [13]. BPSK is represented by the equation

$S_{k}(t)=\sqrt{\frac{2 e_{b}}{T_{b}}} \operatorname{Cos}[2 \Pi f t+\Pi-k \Pi] ; k=0,1$

\section{AWGN channel}

White Gaussian noise is added to real or complex input signals via the AWGN Channel block. A real input signal produces a real output signal by summing together real Gaussian noises. A complex signal goes into this block and it generates a complex output signal by summing complex Gaussian noise. Input signals determine the sample time of this block. OFDM is typically studied with the AWGN channel model. An amplitude distribution of Gaussian noise is added linearly to white noise having a constant spectral density. In addition, there is no consideration for fading, frequency selection and interference. However, it is still recognized as providing simple mathematical models [17].

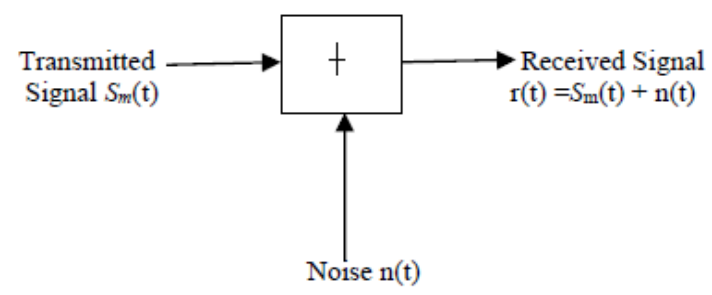

Figure 5. Awgn channel

BER of BPSK Modulation in AWGN Channel : In an AWGN channel, BPSK has a BER of

$$
\begin{gathered}
B E R_{B P S K}=Q\left(\sqrt{\frac{2 e_{b}}{N_{o}}}\right) ; Q(t)=\frac{1}{\sqrt{2 \Pi}} \int_{t}^{\infty} e^{\left(\frac{-y^{2}}{2}\right)} d y \\
B E R_{B P S K, A W G N}=\frac{1}{2} \operatorname{erfc}\left(\sqrt{\frac{e_{b}}{N_{o}}}\right) ; Q(t)=\frac{\operatorname{erfc}\left(\frac{t}{\sqrt{2}}\right)}{2}
\end{gathered}
$$

Here, the complementary error function represented by erfc and $\mathrm{eb} / \mathrm{N}_{\mathrm{o}}$ is the bit energy-to-noise ratio.

\section{E. Rayleigh Fading Channel}

Multipath reception is primarily responsible for Rayleigh fading. Multipath components occur for Rayleigh fading channels, each with random amplitudes and phases. Mobile radio channels commonly use the Rayleigh distribution. Radio signals can be analyzed using this statistical model to assess the affects of propagation environments. This model can be used to represent signal propagation at the troposphere and the ionosphere, as well as the effects of densely populated urban areas on radio signals [18]. Rayleigh distributions are known to govern the envelopes of sums of Gaussian noise signals in quadrature. Time-dependent envelope of Rayleigh distributed signals. Rayleigh fading is of most advantage when the transmitter and receiver are not in line of sight. 
An expression of Rayleigh's PDF is

$$
p\left(r_{o}\right)=\frac{r o}{\sigma^{2}} e\left[\frac{-r_{o}^{2}}{2 \sigma^{2}}\right] ; r_{0} \geq 0
$$

Chi-squared channels distribution gives the PDF of SNR as

$$
\begin{gathered}
p(r)=\frac{e\left(\frac{-r}{r^{\prime}}\right)}{r} ; r^{\prime}=\frac{e_{b}}{N_{o}} \\
B E R_{\text {bpsk,Rayleigh }}=\frac{\left[1-\sqrt{\frac{r^{\prime}}{1+r^{\prime}}}\right]}{2}=\frac{1}{2}\left[1-\sqrt{\frac{e_{b} / N_{o}}{1+\left(e_{b} / N_{o}\right)}}\right]
\end{gathered}
$$

\section{F. Rician Fading Channel}

Radiation propagation is a stochastic process called Rician fading. At least one path changes to enter the receiver as the signal arrives. A line-of-sight path possesses a stronger signal than the other paths leading to Rician fading. A Rician distribution describes the amplitude gain in Rician fading [4]. If there are multiple path locations between transmitters and receivers, Rician fading takes place when a single line of sight communication channel is established.

Fot the received signals, he Rician PDF is

$$
p(r)=\frac{r \cdot e\left(\frac{-\left(r^{2}+a^{2}\right)}{2 \sigma^{2}}\right)}{\sigma^{2}} B_{o}\left[\frac{r a}{\sigma^{2}}\right] ; r \geq 0 ; a \geq 0
$$

Here modified Bessel function is $\mathrm{B}_{0}($.$) and K-factor is$ $K=\frac{a^{2}}{2 \sigma^{2}}$

For Rician channels that are not coherent, the BER is calculated as follows:

$$
B E R_{B F S, \text { Rician }}=\frac{1+\frac{a^{2}}{2 \sigma^{2}}}{2+2 \frac{a^{2}}{2 \sigma^{2}}+\frac{e_{b}}{N_{o}}} \cdot e\left[\frac{-\frac{a^{2} e_{b}}{2 \sigma^{2} N_{o}}}{2+2 \frac{a^{2}}{2 \sigma^{2}}+\frac{e_{b}}{N_{o}}}\right]
$$

If BFSK is not coherent, then the BER in an AWGN channel is

$B E R_{B F S K, A W G N}=\frac{e^{\left[\frac{-e_{b}}{2 N_{o}}\right]}}{2} ; a t, K=\infty$

\section{SIMULATION RESULTS}

We simulate and estimated the bit error rates for various modulation schemes used in OFDM systems.
The Matlab software is an excellent tool for studying the behavior of radio channel characteristics while dealing with different kinds of interference and fading. Figure 6 shows that BER performance of various modulation techniques over a AWGN channel. The figure 7 shows simulated QPSK results over AWGN, Rayleigh, and Rician channels. Simulated BPSK results are shown in figure 8 for AWGN, Rayleigh, and Riccan channels. Over the Rician channel and Rayleigh channel, BPSK modulation has a more promising performance than AWGN modulation. These differences are clear indicators that fading channels perform better than AWGN channels.

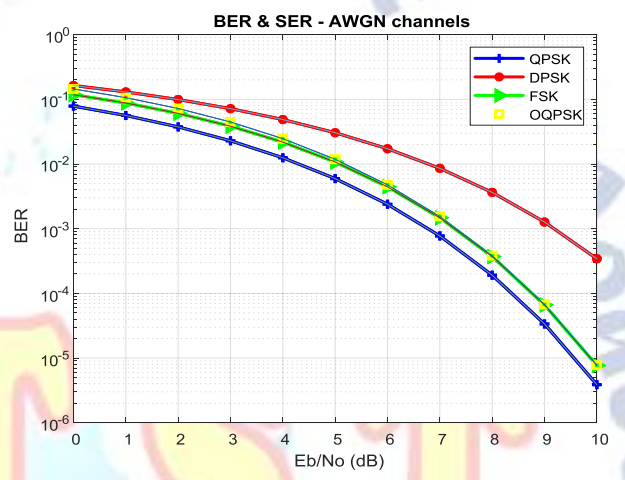

Figure 6 . SER vs BER for AWGN Channel

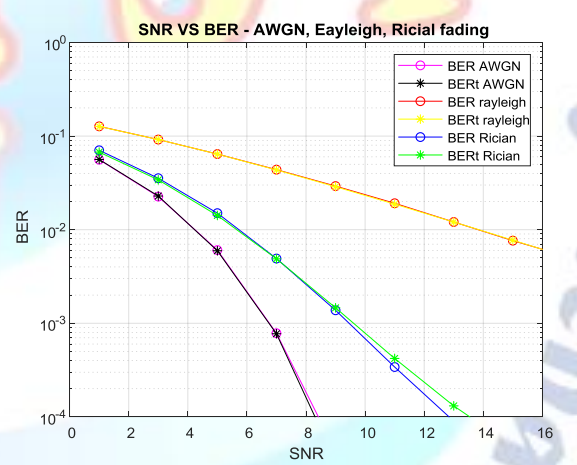

Figure 7. SNR vs BER :QPSK over AWGN, Rayleigh, Rician Fading Channel

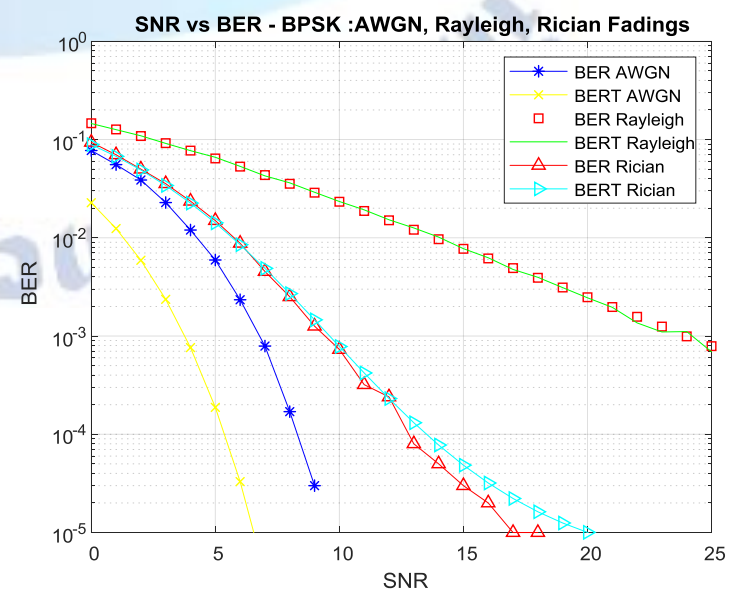

Figure 8. SNR vs BER :BPSK over AWGN, Rayleigh, Rician Fading channel 


\section{CONCLUSION}

Various digital modulation techniques, including multipath fading channels, are examined in this paper. It has been discovered that error rate over AWGN, Rayleigh, and Rician channels improves with modulation order. These experimental results were closely matched with those obtained by theoretical modeling of the link performance for BPSK and QPSK . This paper examines the relationship between BER and SNR.

\section{REFERENCES}

[1] Kommabatla Mahender, Tipparti Anil Kumar and K S Ramesh, "SER and BER Performance Analysis of Digital Modulation Scheme over Multipath Fading Channel", International Journal of Control Theory and Applications, ISSN 0974-5572, Vol 10, pp. 81-91, 2017.

[2] Indu Chandran, M.Raju and K.Ashoka Reddy, "Comparative analysis of various Channel Estimations under different Modulation Schemes", International Conference on Trends in Electronics and Informatics, pp. 832-837, 2017.

[3] Kommabatla Mahender, Tipparti Anil Kumar and K S Ramesh, “ Performance Study of OFDM over Multipath Fading Channels for Next Wireless Communications", International Journal of Applied Engineering Research, ISSN 0973-4562, Volume 12, pp. 10205-10210, 2017.

[4] M.S. Chavan, R.H. Chile and S.R. Sawant, "Multipath Fading Channel Modeling and Performance Comparison of Wireless channel Models", International Journal of Electronics and Communication Engineering, ISSN 0974-2166 Volume 4,pp. 189-203, 2011.

[5] Er. Anil Kumar, Puneet Kumar, Rahul Kumar, Surendra Chaudhary, Vikash Kumar Gautam, "Bits Error Rate Calculation Of BPSK and QPSK Using MATLAB", Journal of Emerging Technologies and Innovative Research, Volume 8, Issue 7, pp. 569-574, 2021.

[6] Rishad Ahmed Shafik, Md. Shahriar Rahman and AHM Razibul Islam, "On the Extended Relationships Among EVM, BER and SNR as Performance Metrics", International Conference on Electrical and Computer Engineering, pp. 408-411, 2006.

[7] M.Raju and K.Ashoka Reddy, "Mean Square Error Analysis in MIMO-OFDM System using Pilot based Channel Estimation", International conference on signal processing communication power and embedded system, IEEE, pp.1632-1636, 2016.

[8] M.Raju and K.Ashoka Reddy, "Evaluation of BER for AWGN, Rayleigh Fading Channels under M-QAM Modulation Scheme", International Conference on Electrical, Electronics, and Optimization Techniques, IEEE, pp.3081-3086, 2016

[9] Upena Dalal, “Wireless Communications, Oxford Higher Education", 2009.

[10] Rappaport, T.S, "Wireless Communications: Principles and Practice". IEEE Press, iscataway (1996).
[11] Hirosaki, b., "An Orthogonally multiplexed QAM system using the Discrete fourier transform", IEEE transaction on communications, Vol.29, pp.982-989, July 1981.

[12] Yong Soo Cho, Jaekwon Kim, Won Young Yang and Chung G. Kang, "MIMO-OFDM Wireless Communications With MATLAB". IEEE,John Wiley \& sons (Asia) Pte Ltd,2010.

[13] Simon, M.K., Alouinii, "Digital Communication Over Fading Channels: A Unified Approach to Performance Analysis". Wiley, New York (2000).

[14] T. Hwang, C. Yang, G. Wu, S. Li, and G. Y. Li, “OFDM and itswireless applications: A survey," IEEE transaction on vehicular technology., Vol. 58, no. 4, pp. 1673-1694, May 2009.

[15] Fleming Frederiksen and Ramjee Prasad, "An overview of OFDM and related techniques towards development of future wireless multimedia communications", IEEE Radio and wireless conference, 2002.

[16] Xiaoyi tang, "Effect of channel estimation error on QAM, BER performance in Rayleigh fading", IEEE transaction oncommunications, Vol. 47, pp. 1856-64, December 1999.

[17] E F Casas and C Leung, "OFDM for data communication over mobile radio FM channels", IEEE Transactions on Communications, Vol.39, No. 5, 1991.

[18] Ming xian chang, "Performance Analysis of Equalized OFDM system in Rayleigh fading channel", IEEE transaction on Wireless Communications. Vol. 1 no. 4, pp 721-732, October 2002.

[19] Karthik Kumar Vaigandla, Mounika Siluveru and Sandhya Rani Bolla, "Analysis of PAPR And Beamforming For 5G MIMO-OFDM", International journal of analytical and experimental modal analysis, Volume XII, Issue X, 2020, pp.483-490.

[20] Hongwei Yang, " A road to future broadband wireless access: MIMOOFDM based air interface", IEEE Communications Magazine, Vol.43, No.1, pp.53-60, Jan, 2005.

[21] H.Sampath, S.Talwar, J.Tellado, V.Erceg and A.Paulraj. "A Fourth Generation MIMO-OFDM broadband wireless system: design, performance and field trail results", IEEE Communications Magazine, No.9, pp.143-149, September, 2002.

[22] G.L.Stuber, J.R.Barry, S.W.Mclaughlin, Y.Li.M.A.Ingram and T.G.Pratt, "Broadban MIMo-OFDM wireless communications",Proceeding of the IEEE, Vol.92, No.2, pp.271-294, February, 2004.

[23] A.Tarighat and A.H.Sayed. "MIMO-OFDM receivers for systems with IQ imbalnces", IEEE Transactions on Signal Processing, Vol.53,no.9, pp.3583-3596, Sep, 2005.

[24] Allanki Sanyasi Rao1, V Karthik Kumar and Malothu Devsingh, "Channel Capacity with and without CSIT for different configurations of MIMO System", The International journal of analytical and experimental modal analysis, Volume XIII, Issue I, 2021, ISSN NO:0886-9367, pp. 48-55, 2021.

[25] Karthik Kumar Vaigandla and Dr.N.Venu, "BER, SNR and PAPR Analysis of OFDMA and SC-FDMA", GIS SCIENCE JOURNAL, Volume 8, Issue 9, 2021, pp.970-977. 